Sociologie et sociétés

\title{
Comment se mobilise-t-on ?
}

\section{L'apport d'une approche pragmatiste à la sociologie de l'action} collective

\section{How do we mobilize? \\ The pragmatist approach in the sociology of collective action}

\section{Daniel Cefaï}

Volume 41, numéro 2, automne 2009

Les mouvements sociaux au-delà de l'État

Social movements beyond the state

URI : https://id.erudit.org/iderudit/039267ar

DOI : https://doi.org/10.7202/039267ar

Aller au sommaire du numéro

Éditeur(s)

Les Presses de l'Université de Montréal

ISSN

0038-030X (imprimé)

1492-1375 (numérique)

Découvrir la revue

Citer cet article

Cefaï, D. (2009). Comment se mobilise-t-on ? L'apport d'une approche pragmatiste à la sociologie de l'action collective. Sociologie et sociétés, 41(2), 245-269. https://doi.org/10.7202/039267ar

\section{Résumé de l'article}

En présentant un ensemble de recherches " pragmatiques » ou " praxéologiques » qui ont eu cours en France depuis les années 1980, et en les augmentant de quelques idées héritées du pragmatisme nord-américain,

l'auteur s'interroge sur leur pertinence pour une sociologie des mobilisations collectives. L'article s'ordonne autour de quatre points.

1. Le développement d'une sociologie des régimes d'action et de justification, d'une part, et de démarches herméneutiques ou grammaticales, d'autre part, complexifie les modélisations structuraliste et rationaliste de l'action.

2. La réactivation de la notion de " public ", empruntée à Dewey, en contrepoint d'une sociologie des problèmes publics, permet de poser des questions sur le politique que les approches en termes de processus, de marché ou de champ politique avaient négligées.

3. L'étude des réseaux et des organisations les conçoit comme des arènes d'expérience et d'action, articulées par des agencements d'objets, dans lesquelles les acteurs s'engagent selon des modalités hybrides. Elle fait apparaître des grammaires plurielles de la communauté, de l'acteur et du collectif.

4. L'approche instrumentale ou stratégique de la culture, entendue comme répertoire de ressources, est enrichie par une conception de la culture publique, centrée sur la formation d'expériences individuelles et collectives. Elle s'appuie sur des réflexions autour des notions d'affectivité et de temporalité.
Ce document est protégé par la loi sur le droit d'auteur. L'utilisation des services d'Érudit (y compris la reproduction) est assujettie à sa politique d'utilisation que vous pouvez consulter en ligne.

https://apropos.erudit.org/fr/usagers/politique-dutilisation/ 


\title{
Comment se mobilise-t-on?
}

L'apport d'une approche pragmatiste à la sociologie de l'action collective

\author{
DAN IEL CEFAÏ \\ Institut Marcel Mauss, EHESS-Paris \\ Université Paris Ouest Nanterre-La Défense \\ 200, avenue de la République \\ 92001 Nanterre cedex \\ Courriel: cefai@ehess.fr
}

\begin{abstract}
Comment SE Mobilise-T-On? Notre hypothèse sera dans ce texte de montrer que la genèse de nouvelles perspectives en sciences sociales en France depuis les années 1980 permet de reformuler un certain nombre de questions dans le domaine de la sociologie des mobilisations collectives. Elle s’appuie sur la multiplicité des expérimentations qui y ont eu cours: économie des conventions, grammaire de la justification et sociologie des régimes d'engagement, anthropologie des sciences et des techniques et des acteurs-réseaux, sciences cognitives, enquête pragmatiste sur les publics, ethnométhodologie, analyse conversationnelle, herméneutique narrative ${ }^{1}$... Si divergentes qu'aient été ces expérimentations - que l'on a trop rapidement rassemblées sous le titre de sociologie «pragmatique» ou «praxéologique» -, elles ont toutes participé d'une refondation - controversée - des sciences sociales en France. Curieusement, la sociologie des mouvements sociaux y est restée assez peu réceptive. Notre objectif n'est pas d'être exhaustif dans le recensement de ces options théoriques et empiriques: le parcours qui est ici présenté est personnel, d'autres sont possibles. Et il serait impossible de tous les explorer dans le cadre d'un article, tout au plus indiquera-t-on un itinéraire de
\end{abstract}

1. Dont on trouve un bon écho dans les livraisons annuelles de la collection "Raisons pratiques", aux Éditions de l'École des hautes études en sciences sociales, Paris. 
lecture. Le lecteur notera en tout cas la place centrale qu'y occupe l'héritage pragmatiste, en particulier celui de John Dewey, mais aussi de William James ou de George Herbert Mead, avec, en arrière-fond, des résonances phénoménologiques. Ce dialogue, qui conduit à réélaborer les thèmes de l'expérience et de la situation, d'une part, du collectif et du public, d'autre part, n'est pas partagé par tous les auteurs qui revendiquent une approche «pragmatique» ou «praxéologique». Notre objectif sera donc de poser quelques questions aux modèles de l'action, de l'organisation et de la culture de l'action collective, tels qu'ils sont élaborés, de façon prédominante en sciences sociales et politiques, en les mettant en regard de ce corpus de littérature, francophone essentiellement, qui a émergé dans les années 1980 et en allant, ici et là, puiser dans l'héritage pragmatiste de nouvelles perspectives de questionnement.

\section{ÉTUDIER DIFFÉRENTES MODALITÉS D'ENGAGEMENT SITUÉ}

Massivement, les théories de l'action rationnelle et de la mobilisation des ressources ont colonisé les raisonnements des sociologues de l'action collective: une version assez pauvre, du reste, qui tendait à rabattre toutes les initiatives de mobilisation collective sur des calculs d'intérêt, matériel ou symbolique. Ressources, contraintes et opportunités ont été transformées en paramètres que les acteurs manient en vue de maximiser l'utilité, l'efficacité et la rentabilité de leurs actions. Gardons-nous d'être simplistes, cependant: les modèles analysant les dilemmes de l'action collective, de Coleman à Axelrod, de Lichbach à Heckathorn, ont une force explicative pour un certain nombre de situations concrètes. Mais dans tous les cas, c'est une rhétorique de l'économie, de l'entreprise et de l'entrepreneur, de la stratégie de rentabilisation des intérêts prédéterminés et des capitaux disponibles qui l'a emporté et qui a colonisé cette littérature.

De ce point de vue, qu'a-t-on appris à la faveur des nouvelles sociologies de l'action?

a) Progressivement, le répertoire des modélisations de l'action a été enrichi. D'autres modes d'engagement, formes d'expérience et régimes d'action se sont imposés à la recherche. Après sa rupture avec Touraine, pour qui la question de la culture et de l'identité des NMS était centrale, Quéré a prospecté du côté de l'agir communicationnel de Habermas (1987) (qui s'était lui-même inspiré, entre autres, de Mead) et a travaillé sur la sémantique et l'herméneutique de l'action selon Ricœur $(1977,1981)$ et sur les procédés de catégorisation des ethnométhodologues (Garfinkel, 2007). Que l'on prenne en compte les conditions d'une délibération publique en vue d'atteindre des solutions raisonnables, les activités de configuration narrative des actions ou les activités des membres pour coproduire un sens situé, on a clairement affaire à des façons de se rapporter à la réalité et de façonner des rationalités et des légitimités que les sociologies de l'action collective ont peu prises en compte — sinon récemment, dans des tentatives de recroisement avec les approches de démocratie délibérative. De nombreux débats sur l'action, empruntant autant à Wittgenstein qu'à Davidson, à Von Wright qu'à Apel, ont battu leur plein en France à la fin des années 1980. Cette reprise des questions de la philosophie analytique et de la philosophie pratique a impulsé différents 
programmes de sociologie morale, comme, au Centre de sociologie de l'éthique, celui de Pharo (1985), qui s’interrogeait sur la logique déontique des actions - leur qualification en termes d'interdit, de facultatif, d'autorisé et d'obligatoire - , conduisant à des formes de désapprobation, de dénonciation, de revendication et de mobilisation, soit à l'émergence d'un sens civique. Quéré prenait ses distances avec la notion de mouvement social et prônait une praxéologie de l'opinion publique (1990), rejoignant bientôt l'interrogation de Descombes sur les collectifs et réarticulant l'enquête sur les mobilisations autour de l'expérience de problèmes publics.

b) De leur côté, Boltanski et Thévenot fondent en 1985 le Groupe de sociologie pragmatique et morale (GSPM). Leurs efforts se concentrent sur un projet d'enquête grammaticale sur les formes de justification, de dénonciation ou de revendication en public (Boltanski, 1984). De la justification (Boltanski et Thévenot, 1991) propose une sociologie des épreuves et fournit des outils de description et d'analyse des actions en public. Leur typologie des cités - domestique, industrielle, marchande, civique, de l'inspiration et de l'opinion — a pu être critiquée parce qu'elle figeait l'indétermination des situations dans un corset structural et qu'elle se prêtait à des usages dogmatiques. Mais plutôt que d'arrêter une typologie des cités, De la justification invite à enquêter sur des moments d'épreuve, où est rendue sensible la complexité des contextes d'expérience et d'action. Dans ces moments de tension, de conflit ou de crise, autour de situations problématiques, se mettent à nu et se donnent à voir des opérations d'ajustement à une réalité et de coordination des tâches. Dans cette perspective, les acteurs n'obéissent pas à une logique d'action: dans leurs opérations d'appréciation et de jugement sur «l'action qui convient» (Thévenot, 1990), ils agissent «dans plusieurs mondes» (Dodier, 1991), entre lesquels ils basculent ou qu'ils composent les uns avec les autres. Ils le font en trouvant des appuis pragmatiques (Dodier, 1993) dans leurs environnements afin d'agir de façon pertinente, cohérente et conséquente. Corcuff (1991) avait appliqué cette perspective à une enquête sur le syndicalisme cheminot.

c) La question de base ne porte plus alors sur les «déterminants structuraux» et les «calculs rationnels» de l'action: elle substitue à la platitude de l'action instrumentale des architectures complexes de la situation, de la personne et du collectif. Le pluralisme des ordres normatifs dans le régime de justice s'est redoublé d'un pluralisme des régimes d'action: le régime de justice (qui fonde une politique justifiée) côtoie, dans L'Action au pluriel de Thévenot (2006), un régime de l'action familière (qui fonde une politique du proche) et un régime de l'action en plan (qui fonde une politique rationnelle). Les chercheurs du GSPM ont alors exploré d'autres régimes d'expérience et d'action - celui de la compassion dans la médiatisation de l'humanitaire et celui des situations-limites, hors justification, de l'agape, de l'amour et de la paix entre personnes singulières ou, à l'autre bout du spectre, du rapport de force et de la loi du plus fort, ont été explorés par Boltanski (1990, 1993; Boltanski et Chiapello, 1999). Par ailleurs, les étudiants de Thévenot se sont engagés dans une enquête sur les "politiques du proche», réhabilitant des formes d'engagement familier dans le monde, souvent discréditées en France au nom de l'intérêt général (Trom, 1999; Ion, 2001) — et ques- 
tionnant la conception républicaine de la citoyenneté. Comment les attachements que l'on dit «familiers», «personnels» ou « intimes» contribuent-ils à «faire du commun» et à «faire du public»? Outre le travail novateur de Stavo-Debauge sur les catégorisations ethniques, ont écrit des thèses éclairantes, de ce point de vue, Auray (2001) sur l'hacktivisme et le mouvement du logiciel libre, Doidy (2002) sur le conflit d'aménagement autour du tunnel du Somport et sur droit au logement. Lafaye a proposé un papier de synthèse sur les ancrages de proximité et les contestations de l'action publique (2006).

Hétérogénéité des régimes d'action, donc, qui enrichit les thèses de la détermination structurale et du choix rationnel. Les acteurs ne sont plus seulement des marionnettes mues par des conditions objectives auxquelles ils obéissent, ils ne sont plus seulement des petits démiurges qui calculent en termes de rentabilité ou de productivité. Ils sont confrontés à des mondes, dont ils éprouvent la réalité et la justice, en relation à leurs manières de s'y engager; ils sont donc soumis à des contraintes écologiques fortes, tout en disposant d'une marge de manœuvre qui leur ménage une liberté. Et surtout, ils disposent d'une capacité de jugement esthétique, moral et politique (pour un panorama GSPM: Pattaroni, 2001 et 2005). Leur enquête est du coup réceptive aux engagements de devoir, de responsabilité et de solidarité, aux soucis de soi et d'autrui et aux idéaux de «bien vivre ensemble» qui se jouent dans des actions collectives — tout comme elle l'est aux «impératifs de justification» de l'action en public (Pharo, 1985; Quéré, 1990; Boltanski et Thévenot, 1991).

\section{INTRODUIRE LA QUESTION DU «PUBLIC EN CONTREPOINT DE CELLE DU PROCESSUS POLITIQUE}

La reprise de la perspective pragmatiste est venue se greffer sur ces interrogations, au moins pour quatre raisons. On trouve en effet chez James, Mead et Dewey des conceptions fortes de l'expérience et de l'action; ils ont tenté d'élaborer une réflexion qui parte de l'expérience de situations en situation et non pas de modèles intellectuels; Mead et Dewey n'ont jamais dissocié la problématisation de l'action d'une réflexion sur leurs engagements civiques et politiques; enfin, Dewey a forgé un concept de public, qui est revenu récemment au centre des débats (Zask, 2003; Cefaï et Pasquier, 2003; Stavo et Trom, 2004; Latour, 2008). Si l'on s'en tient à ce dernier point, la question du public nous semble soulever des problèmes qui sont absents des théories du processus politique.

a) Une démarche pragmatiste se situe dans un autre registre que celle du processus politique. Au lieu d'avoir une vision en simple extériorité par rapport à ce qu'elle décrit, elle accompagne les «expériences» et les «perspectives» des acteurs. En s'inspirant de l'héritage de Park, Mead et Dewey, elle part de l'idée qu'une dynamique de mobilisation collective est un processus de co-définition et de co-maîtrise de situations problématiques. L'enjeu est de décrire la mutation des expériences collectives et des environnements institutionnels en les faisant émerger depuis les activités en train de s'y accomplir. Comment la trajectoire d'un problème public ordonne-t-elle un horizon d'engagements, de concernements, de sensibilisations et de mobilisations à ses 
entours? Quels procès d'association, de coopération et de communication se constituent autour de ce problème public? Quels montages institutionnels, juridiques et politiques vont éventuellement donner une solution à ce problème public? Comment des arènes publiques s'articulent-elles autour de dynamiques de constitution de problèmes publics, auxquelles participent les mobilisations collectives? La question est moins celle du "public et de ses problèmes» que du «problème et de ses publics» ${ }^{2}$. Dans ces processus de publicisation, les individus sont souvent découplés des rôles et des statuts, des opinions et des convictions qui sont les leurs d'ordinaire; et les logiques d'action débordent les marchés, les champs ou les secteurs où elles sont contenues d'ordinaire, en passant par-dessus leurs frontières, par un phénomène que les théories $\mathrm{du}$ comportement collectif qualifiaient de contagion ou de propagation. Le public redéfinit l'horizon des possibles, plutôt qu'il n'est contraint par des structures d'opportunité politique. C'est ce que nous avons appelé ailleurs une «arène publique» (Cefaï, 2002).

b) Ces questions rebondissent sur une nouvelle: qu'est-ce qui est politique et qu'est-ce qui ne l'est pas? Une démarche pragmatiste nous semble en excès sur la définition que donnent McAdam, Tarrow et Tilly (2001: 5) ou Tilly (2003) de la «politique conflictuelle ${ }^{3}$.» L'horizon du vivre-ensemble et du bien vivre ensemble ne cesse de se reconfigurer à l'épreuve des actes de résistance et de protestation, de désobéissance et de critique, de dénonciation et de revendication, ou tout simplement, sans se charger nécessairement de cette valence négative, d'enquête et d'expérimentation, de coopération et d'innovation. Les bornes de ce qui est «politique» et de ce qui ne l'est pas en sont sans cesse déplacées - en particulier celles du "personnel» et du «politique». Par ailleurs, la reconnaissance d'une multiplicité de «sphères de justice» par Walzer avait trouvé un prolongement dans la typologie des cités de Boltanski et Thévenot (1991): on découvrait que de multiples grammaires de justification pouvaient se composer dans des revendications collectives, certaines a priori antithétiques d'un régime civique-républicain. Depuis, un certain nombre de programmes de recherche, qui ont puisé tant dans la phénoménologie que dans le pragmatisme - sur les politiques du proche (Thévenot et Breviglieri, 2009), sur l'esthétisation des espaces publics (Lolive, 2006) ou sur le militantisme des experts naturalistes (Micoud, 2007) — ont montré comment des actions collectives, articulées autour d'engagements touchant à la sensibilité, dessinent de façon neuve des figures de la res publica. Sur une autre ligne, approfondissant les éco-politiques, qui mettent l'environnement au centre des politiques publiques, allant jusqu'au bout de la symétrisation entre humains et non-humains, les cosmopolitiques de Latour (1999) ont rapatrié la nature au cœur de la polis. On a là une multiplicité de thématiques qui toutes font écho à des formes émergentes d'expérience du politique.

2. Cette formulation est due à une discussion avec Louis Quéré et Cédric Terzi.

3. La politique conflictuelle consiste en une activité de revendication, collective et publique, dans un processus dont l'une des parties est nécessairement l'État — défini comme une organisation «qui exerce le contrôle sur la plus forte concentration de moyens de coercition sur un territoire». 
c) Dans le même mouvement, le pouvoir n'est pas seulement entendu comme rapport de domination et de subordination, ni de normalisation et de résistance. Il est aussi compris comme puissance d'institution de capacités et de droits (du côté de l'État) et comme puissance d'agir de concert (du côté du public). Les dispositifs d'action publique (Trom et Laborier, 2003), par exemple, ne font pas qu'enserrer des «sujets» dans des dispositifs d'assujettissement, de discipline, de contrôle et de normalisation. Ils instaurent de nouveaux droits et délèguent de nouveaux pouvoirs, corrigent et réparent des injustices de traitement, œuvrent à restituer à chacun des chances équitables de réussite. La potestas in populo, qu'Arendt (1972) identifiait dans les mobilisations du mouvement des droits civiques, cet exercice d'un pouvoir collectif, qui bouleverse des environnements techniques, juridiques, institutionnels et politiques, se redouble d'une forme d'empowerment, capacitation faite d'apprentissage de l'autonomie, d'accès à la dignité et à la responsabilité, et de reprise du contrôle sur sa propre vie (De Munck et Zimmermann, 2009). Les enquêtes de Barbot (2002) sur les associations de lutte contre le sida ou de Callon et Rabeharisoa (1999) sur l'Association française contre les myopathies ont montré comment le statut du patient et l'expérience de la maladie se sont transformés dans le cours de ces mobilisations collectives. Les expérimentations des forums hybrides, même si elles ne se portent pas toujours sur le terrain décisionnel, s'articulent souvent avec des entreprises associatives et restituent aux citoyens une part de délibération jusque-là réservée aux élus et aux experts (Callon, Lascoumes et Barthe, 2001). Bien sûr, tout n'est pas si rose et nombre de mobilisations, sans aller jusqu'à défendre des causes antidémocratiques, peuvent conduire à des formes de blocage des institutions démocratiques, et saper les mécanismes de régulation ou de représentation (Rosanvallon, 2006). On a en tout cas là un certain nombre d'éléments qui imposent de transformer les thèses dominantes sur le processus politique.

\section{SAISIR LES RÉSEAUX ET LES ORGANISATIONS COMME DES CONTEXTES D'EXPÉRIENCE ET D'ACTION EN TRAIN DE SE FAIRE}

Les organisations de mouvements sociaux ont longtemps été traitées comme des «infrastructures matérielles de mobilisation», des machines de guerre plus ou moins efficaces contre l'adversaire ou des gisements de capital social à investir et à rentabiliser. Cette vue s'est complexifiée avec le temps. Elles sont aussi des milieux de sociabilité, où émergent des occasions de rencontre et qui façonnent des formes de coexistence. Elles sont des agencements d'objets, de normes et de personnes qui ordonnent ce que les membres peuvent faire, voir ou dire. Elles constituent des environnements praticosensibles, qui fixent des habitudes de coopération et de conflit et qui fournissent des repères d'expérience cognitive et normative ${ }^{4}$. Elles sont indissociablement des vecteurs

4. Voir par exemple le concept de «styles de groupe» de Eliasoph et Lichterman (2003) et Lichterman (2005), qui a mis l'accent sur les «activités de faire sens» (meaning-making activities). Il rend compte d'une «culture» en action et en interaction, dont les normes discursives, les liens internes et les frontières externes sont toujours en train de se faire et qui permet de reconnaître des manières typiques de comprendre, d'interagir ou de communiquer. 
de concentration de capitaux matériels et humains, des incubateurs de réseaux d'activistes, des couveuses d'entreprises militantes, des générateurs d'énergie symbolique, des instances de représentation collective. Un certain nombre de travaux dans ce domaine d'étude peuvent être mis en dialogue avec une approche plus pragmatique et pragmatiste.

On peut d'abord penser les organisations comme des regroupements, plus ou moins fluides, autour de situations problématiques à résoudre, qui mettent en jeu une «expérience créatrice» — une idée anticipée par des pragmatistes comme Follett dans The New State (1998). En tentant de trouver des solutions, au cours du temps, elles se transforment en interne ou transforment leur environnement. Il se peut qu'elles puisent dans des «répertoires de formes organisationnelles disponibles» et qu'elles s'en inspirent pour créer un nouveau type, comme Clemens (1997) l'a montré à propos des organisations de suffragettes qui ont hésité entre les options du club, du parlor meeting, de la charitable society et du parti politique. Elles expérimentent des schémas de circulation des informations, de répartition des pouvoirs et de division des tâches, de coopération entre opérateurs, de distribution des droits et de concentration des décisions. Mais en faisant ces choix organisationnels, elles déterminent aussitôt le type d'expériences et de revendications, d'alliances et de conflits, d'actions et de marges de manœuvre qu'elles vont avoir. Il en allait ainsi avec la mise en œuvre de principes de démocratie participative par les organisations de la New Left dans les années 1960, étudiées par Polletta (2002). Les organisations ont par ailleurs de plus en plus le statut de nœuds de «réseaux d'interactions informelles entre une pluralité d'individus, de groupes ou d'associations, engagés dans un conflit culturel ou politique, et partageant une identité collective» (Diani, 1992: 13). Friedberg (1992) a proposé de parler plutôt d' "actions organisées", résultantes instables d'interactions en prise sur un "ordre local», dont les membres poursuivent des finalités non nécessairement compatibles, sont pris dans des tensions entre objectifs intermédiaires et ultimes, entre grandeurs d'échelle territoriale ou temporelle, ou entre segments locaux, ethniques ou professionnels. Dans ces «actions organisées » s'entrelacent divers processus de coordination et de compétition autour de la définition et de la solution aux problèmes rencontrés, des compromis instables sont trouvés autour de formules stratégiques et de conventions collectives (Orléan, 1994) et des régimes d'engagement, des rationalités et des légitimités multiples se composent (Thévenot, 1993, 2001, 2006).

Une autre idée, défendue en premier lieu par Gusfield (1981), dans la tradition de Park et Blumer, est que ces organisations doivent être resituées dans des environnements «fluides» et "diffus», faits de chaînages d'interaction entre contextes d'expérience, engendrant d'imperceptibles mutations des mours et des croyances, plutôt qu'autour des seuls axes «linéaires» de stratégies organisationnelles, orientées vers leurs objectifs et mesurant succès et échecs à l'aune de l'efficacité de leurs coups. La modélisation des organisations de mouvements sociaux comme entreprises, à l'œuvre sur le marché de la protestation sociale, avait négligé les recherches sur le comportement collectif - courants d'opinion, modes ou rumeurs - auxquelles Gusfield se réfère. Là encore, ces idées ont fini par faire leur chemin, contre les résistances des approches 
structurales et rationnelles. L'analyse de réseaux a transformé la vision que l'on avait des «structures de mobilisation» et ouvert des pistes pour explorer ces processus de diffusion. Dès les années 1970, elle diagnostiquait que certains mouvements sociaux n'étaient plus tant enserrés dans des organisations centralisées et hiérarchisées, que réticulaires, acéphales ou polycentrées. Melucci (1996) a poursuivi dans les années 1980 : les mutations des «aires de mouvement» montraient le brouillage des identités collectives et la désaffection des organisations militantes dans un monde postmoderne. Il décryptait les «défis symboliques» aux «codes culturels» qui selon lui fondaient une autre politique, plus centrée sur des enjeux de la vie quotidienne et sur les «jeux du Soi». On pourrait lire les travaux plus récents de Ion (2001) sur l'engagement « distancié» ou «affranchi» ou ceux de Boltanski et Chiapello (1999) sur la «critique artiste» dans un monde connexionniste en écho lointain à ces perspectives et en rupture avec elles.

Une voie intéressante pour une approche pragmatiste est celle empruntée par Mische (2008), qui semble répondre à la critique relationnelle et culturelle à l'analyse structurale des réseaux de Emirbayer et Goodwin (1994). Mische donne corps à une vision deweyenne des publics. Les réseaux sur lesquels elle a enquêté se reconfigurent à travers des ruptures dans des équilibres situationnels. Ils peuvent être bousculés par des événements qui viennent défaire et refaire les liens qui les composent; ils se reforment autour de dynamiques de définition et de résolution de situations problématiques. Mische a montré, dans son enquête sur les réseaux d'organisations lors des luttes pour l'impeachment de Collor de Mello en 1992, au Brésil, comment des fronts d'alliance et d'opposition n'ont cessé de se décomposer et de se recomposer. Ces acteurs coalisés dans des «publics partisans» doivent riposter à des défis de leurs adversaires, à leurs stratégies de déstabilisation et de division, contrecarrer les opérations de contreinformation, de critique ou de discrédit qui les visent. Ils doivent faire face à des insatisfactions de la base, rectifier le tir après des renversements d'alliance et anticiper les conséquences de leurs choix stratégiques. Ils doivent prendre acte de rapports d'enquête qui changent la définition de la situation, rompre ou renforcer des liens en fonction de la capacité de leurs porte-parole à être sur la même longueur d'onde, réussir à surfer sur une vague de sympathie inattendue que déclenche leur action. Ils doivent produire des plateformes programmatiques qui permettent à des acteurs aussi différents que des clubs de patrons, des associations d'étudiants, des syndicats marxistes ou des coordinations catholiques d'y prendre part... Les configurations de prises de position publiques passent par des opérations de «découplage» des acteurs de leurs réseaux de "positions» et par le remodelage de leurs paysages organisationnels, lesquels se temporalisent corrélativement aux débats qui fixent des lignes revendicatives et aux événements qui n'ont cesse d'ébranler ces réseaux anti-Collor.

On a là déjà un bel ensemble de questions, issues de ces domaines si novateurs que sont la sociologie des organisations et l'analyse des réseaux — on n'insistera pas sur leurs affinités possibles avec une théorie du "public». Qu'ont apporté de neuf les nouvelles sociologies de l'action sur ces questions-là? Énonçons trois points. 
a) Organisations et réseaux préexistent à l'action collective et qui la déterminent, sont des composantes de l'agencement qui la caractérisent. Le concept d' "agencement» est ici important. L'action collective est vertébrée par des agencements comportant des personnes et des objets, des outils et des récits, des rituels et des symboles; elle est armée par des routines et des habitudes, ordonnées par des divisions du travail, des répartitions du pouvoir et des distributions du savoir, fixées dans des environnements d'objets, des dispositifs sociotechniques et des circuits opérationnels. On ne peut donc pas s'en tenir à des analyses en termes de consensus des représentations (l'harmonie des convictions idéologiques), de congruence des motivations (la compatibilité entre des mobiles psychologiques) ou d'agrégation des engagements (la synchronie des intentions stratégiques) pour rendre compte de la constitution de collectifs. De ce point de vue, l'apport des sciences cognitives sur la place des objets (Dodier, 1995; Conein et Thévenot, 1997) a parachevé la remise en cause par la philosophie de la place démesurée accordée aux sujets, individuels et collectifs. L'action collective est aussi agie par ses agencements. La notion d'agencement est particulièrement opérante dans les mobilisations sociotechniques. Les réseaux de veille et d'alerte décrits par Chateauraynaud et Torny (1999) sont des actants à part entière, qui émergent et se stabilisent à travers des processus de définition et de résolution de situations problématiques, comme les risques de séisme ou d'inondation. Les coquilles Saint-Jacques, les pêcheurs et les scientifiques étudiés par Callon (1985) coopèrent dans des stratégies de mobilisation en vue du développement économique dans la baie de Saint-Brieuc. Les hacktivistes de Auray (2002) fomentent des subversions techniques, éthiques et politiques, en collaborant dans des communautés virtuelles avec leurs équipements et leurs logiciels. L'«intelligence organisée», comme écrivait Dewey (2003), est ce qui soutient, distribue et configure l'expérience des acteurs, ce qui commande aussi aux modalités d'engagement dans l'action collective et ce qui pérennise les inventions et les émergences de la vie des publics.

b) Deuxièmement, de même que l'on a parlé de feuilletage des régimes d'action et de justification, de même peut-on explorer le feuilletage des trames réticulaires ou des montages organisationnels, en telle ou telle situation. Les «collectifs» sont «brouillés», «composites», «hybrides» ou «mixtes» (Thévenot, 1993 et 2006; Dodier, 1991 et 1993; Lafaye, 1996; Dupuy et Livet, 1997). Sans doute, ils fonctionnent sur des modes qui peuvent être familiaux, entrepreneuriaux, bureaucratiques, ethno-religieux ou civiques... et se rapporter à d'autres principes, éthiques et politiques d'organisation. Plusieurs dynamiques d'action s'entrelacent selon qu'un "collectif» agit comme une entreprise lucrative sur un marché de biens et services, engagée dans une compétition pour le profit, dépendante d'une clientèle d'acheteurs; ou comme une agence publique, obéissant à des règles institutionnelles, légales, statistiques et administratives, œuvrant pour le bien-être de ses usagers. Ce «collectif» peut encore faire office de milieu de sociabilité, produisant de l'entre-soi, facilitant les rencontres, catalysant les sympathies et garantissant des solidarités, forgeant des expériences communes qui peuvent aller jusqu’à la «communauté de destin». Il peut scander la vie quotidienne par des rituels, 
commander à l'élection des affinités, à la mise en scène de soi, des relations avec les autres et des lieux de vie et dicter ce qu'il est bon ou mauvais de croire, de dire et de faire. Ce «collectif» peut enfin se transformer en pseudo ou crypto-parti, en s'assurant de la loyauté d'élus et en noyautant un parti existant ou en fondant son propre groupement politique; ou encore, il peut prétendre incarner une inspiration divine, défendre la religion vraie, poursuivre l'accomplissement d'une prophétie, agréger des fidèles enthousiasmés par une promesse de salut, au nom d'une théologie de la protestation ou de la prospérité, et fonder une église nouvelle. Mais plutôt que de fixer des typologies d'organisations, une démarche pragmatique ou praxéologique centrera le travail descriptif et analytique sur des modalités d'engagement dans des situations. Elle montrera, par exemple, comment certaines fractions du mouvement évangéliste au Brésil ou islamiste en France «composent» entre différents régimes d'action, ségrégant leurs publics, tenant des doubles ou des triples langages, gérant des tensions au sein de leurs personnels et assurant des équilibres entre moyens et finalités... La pragmatique des régimes d'action est une incitation à développer l'effort de décrire et d'analyser, en situation, l'embrouillamini de logiques de rationalité et de légitimité qui s'imbriquent dans des cours d'action et de déchiffrer les modes d'organisation de l'expérience qu'en ont leurs acteurs et leurs spectateurs.

c) Enfin, il existe une pluralité de grammaires du Je, du Tu et du Nous, de façons de faire les collectifs et de les représenter — qui changent d'une société civile et politique à l'autre. Quand on parle d'arènes publiques, on est en droit d'imaginer un espace accessible à tous, sans restriction, et inappropriable par personne, par opposition à un espace communautaire, où des acteurs s'apparaissent les uns aux autres et se révèlent, se découvrent et se transforment au risque de leur exposition publique. On peut encore concevoir un «lieu de l'action collective» (Quéré, 1992, 1995 et 1996; voir aussi Joseph, 2007) où les conditions transcendantales d'égalité et de pluralité sont plus ou moins respectées et dont les protagonistes se battent en vue de biens publics, au nom de l'intérêt général. Mais les choses sont sensiblement plus compliquées dans les faits. Si l'on ne veut pas stigmatiser le particularisme, le corporatisme ou le communautarisme des actions collectives, dans des mondes russes, chinois ou brésiliens, ou tout simplement, dans des mondes professionnels, ethniques ou religieux, à côté de chez soi, il est nécessaire de suivre minutieusement, sans a priori normatif, les chaînages d'activités qui conduisent à la formation de collectifs, de problèmes et de causes. Les dynamiques de «mobilisation», corrélatives à des dynamiques de "problématisation» et de "publicisation », mettent en œuvre des régimes d'action et de justification dont la commensurabilité ne va pas de soi. Les catégories du privé, du commun et du public, du personnel et du politique ne sont pas exportables comme telles, et quand elles paraissent l'être, elles peuvent cacher de faux amis ${ }^{5}$. Un exemple parmi beaucoup d'autres qui impose

5. Thévenot a engagé ce type de programmes de comparaison avec la Russie et avec les États-Unis: voir le résultat d'une coopération de chercheurs français et états-uniens in Lamont et Thévenot (2000). Mais tous les travaux d'anthropologie, de sociologie ou de politique comparée se heurtent aux mêmes problèmes de traduction. 
de la prudence méthodologique: l'usage de l'expression «organisations communautaires », commune aux États-Unis, au Québec et au Brésil, renvoie à des modalités d'être-ensemble, d'identifier des individus et de s'associer en collectifs, de faire du commun, de transiger avec des pouvoirs, de porter des intérêts, de se référer à des droits, de demander des comptes et de rendre des comptes, qui ont peu à voir les unes avec les autres. Toutes les enquêtes sur la constitution d'une "société civile mondiale», qui somment des organisations associatives de toutes sortes afin de mesurer et comparer entre les «tiers-secteurs» de différents pays, passent outre ces distinctions grammaticales - et finissent par additionner des carottes et des navets. L'enquête doit cultiver une sensibilité interprétative et pragmatique aux idiomes de l'engagement, et aux dispositifs politiques, juridiques, institutionnels, théologiques, administratifs d'une grande hétérogénéité auxquels ils réfèrent. En contrepoint, elle investigue comme Lichterman (1996) sur les formes thérapeutique ou religieuse de la «politique personnelle» aux États-Unis ou comme Gonzalez (2009) sur les processus d'individuation et de communautarisation dans les mobilisations évangéliques en Suisse, faisant apparaître des figures variées de la personne et de ses droits, des partages de ce qui relève du public et du privé, et des façons de concevoir la communauté — citoyens, habitants ou croyants. Cette approche grammaticale nous rend sensibles à la multiplicité de ce qui fait «intérêt» dans une action collective: ce qui tient les gens ensemble, ce à quoi ils tiennent et ce qui les fait tenir.

\section{ROMPRE AVEC UNE VISION INSTRUMENTALE ET STRATÉGIQUE DE LA CULTURE PUBLIQUE ET DE L'EXPÉRIENCE COLLECTIVE}

Passons rapidement, pour commencer, sur le refoulement dans les années 1970 de la question du sens, centrale dans l'étude du comportement collectif (collective behavior) des héritiers de la tradition de Chicago, attentifs aux symboles, imageries, identités et émotions (Cefaï, 2007). Le fétichisme d'un Homo oêconomicus a alors court-circuité toute dimension culturelle - qui semblait le privilège des "Européens», Touraine, Offe ou Melucci. Celle-ci a reparu dans les années 1980 aux États-Unis. Un domaine de la sociologie culturelle (cultural sociology) s'est développé. Pour les uns, il s'agit de découvrir des «structures culturelles», en suivant la voie de Durkheim ou Parsons (Alexander) ou de Bourdieu (DiMaggio ou Lamont). Pour d'autres, parfois les mêmes, c'est une conception stratégique de la culture comme boîte à outils (Swidler) qui a permis de contrer les visions trop déterministes du culturalisme. Mais c'est la frame analysis de Snow et de ses étudiants qui a conquis une place forte dans l'étude des mouvements sociaux - au prix d'une transformation de la notion goffmanienne de «cadre» en répertoire de ressources cognitives, où les leaders d'organisations vont piocher pour monter des stratégies de communication dans le jeu de leurs alliances et de leurs oppositions (Cefaï, 2001, 2002 et 2007). Cette vision des choses a suscité une polémique dans le domaine de l'étude des mouvements sociaux, dont l'un des points culminants a été l'article de Jasper et Goodwin (1999), qui attaquait ouvertement les raccourcis structuraux et stratégiques de la théorie de la mobilisation des ressources et 
du processus politique. Depuis, de nombreuses perspectives d'enquête ont émergé, qui recourent à des notions empruntées à la narratologie, à la dialogique ou à la rhétorique. Plus récemment, Eliasoph et Lichterman (2003) ont développé une conception plus située de la culture, comme fabrique de sens en action et en interaction, et sont entrés en dialogue avec les travaux français de sociologie pragmatique.

$\mathrm{Au}$-delà de ces analyses, on peut également tenter, dans une perspective pragmatiste, de forger un concept d'expérience, qui inclue les dimensions de l'affectivité et de la sensibilité, de la mémoire, de l'imagination et du projet. L'expérience est souvent indûment rabattue sur le pôle «subjectif» de l'action. Mais selon James (2007), elle précède la bifurcation entre sujet et objet, et elle est selon Dewey (1993) une transaction entre des organismes et entre ces organismes et leur environnement qui a la valeur d'une épreuve: elle transforme simultanément la situation qui est soumise à l'épreuve et les sujets qui subissent l'épreuve. L'expérience est eue dans un mixte de pâtir et d'agir (Quéré, 2002). Notre hypothèse est que l'enjeu principal de nombre de mobilisations collectives est d'engager le devenir collectif et public d'un flux d'expérience. Quand Dewey (2003) décrit l'émergence du collectif d'explorateurs, d'enquêteurs et d'expérimentateurs qui forme un public, il fait référence à cette dimension expérientielle, indissociablement affective, cognitive et normative, ancrée dans le présent, mais ouvrant sur des horizons de passé et d'avenir, où des convictions «personnelles» vont se former, se renforcer et s'exprimer dans un procès de «collectivisation» et de «publicisation» d'une affaire, d'un problème ou d'une cause. L'expérience collective et publique qui en découle n'est pas la somme ou l'intégration d'une série d'expériences individuelles et privées. Le propre d'une mobilisation qui n'est pas toute de routine est qu'elle ébranle le consensus présomptif de l'expérience de sens commun et qu'elle met à l'épreuve l'attitude naturelle des individus qui la composent. Elle doit produire alors les termes de son intelligibilité et de sa légitimité pour les auditoires auxquels elle s'adresse, pour les membres qui la composent et pour les adversaires contre qui elle se bat. La constitution d'un enjeu de mobilisation, de protestation et de conflit va de pair avec l'articulation d'une arène publique, où pointent des acteurs collectifs et où émergent de nouveaux univers de sens — sens commun, même si sens controversé.

Qu'est-ce que cela veut dire plus précisément? Quand des «individus» se font «consommateurs», «citadins» ou «citoyens», «femmes», «sans travail» ou «sanspapiers», à travers leurs dénonciations et revendications, ils doivent rendre compte (account) de ce qu'ils font, à cause de quoi ils le font et en vue de quoi ils le font et rendre des comptes. Un certain nombre d'activistes ont œuvré à la constitution d'un langage commun, à l'énonciation de répertoires d'identité collective et à la confection de modes d'engagement public. La tâche des premières féministes a ainsi été de découvrir des points communs à la situation des femmes, de donner à des troubles privés une portée publique et de convertir des malaises personnels en cause collective. Les activistes qui viennent ensuite n'inventent pas de but en blanc les rôles qu'elles jouent, mais habitent des univers de sens qui leur sont légués, les réaménageant à la faveur des nouveaux défis qui se présentent. De même, des conflits sont identifiables comme relevant du 
mouvement des sans-papiers parce qu'ils réactivent une expérience collective et publique qui a sédimenté dans des actions précédentes. Ils remanient cette expérience à l'épreuve des péripéties des actions en cours, l'augmentent par des enquêtes, des expérimentations et des discussions, l'enrichissent par des efforts analytiques ou esthétiques, inventant de nouveaux formats d'action si la nécessité s'en fait sentir. Ce que l'on appelle la «culture publique ${ }^{6} »$ est le fonds commun qui donne sa forme et son matériau aux mobilisations collectives. Cette culture publique peut être mise en veille ou «déborder» d'un mouvement à l'autre (Taylor, 2005) — plus justement, être transmise et apprise, reprise et distordue, appliquée et recréée, détournée ou sanctifiée, appropriée et réinvestie de sens neuf. Elle se joue dans un travail de coopération et de compétition où, corrélativement à la défense de leurs intérêts et à la revendication de leurs droits, des acteurs délibèrent rationnellement et raisonnablement, configurent des explications et des interprétations, mais aussi, expriment et symbolisent des émotions, projettent des imaginaires et des utopies.

L'action collective s'organise donc en organisant ses environnements, et produit simultanément les critères d'expérience qui vont permettre à ses acteurs de s'y orienter, de comprendre ce qu'ils y font et ce que les circonstances font d'eux. Ce faisant, ils contrôlent tant bien que mal les situations auxquelles ils ont affaire, moyennant des processus d'enquête, d'expérimentation et de délibération; et ils pilotent les opérations de coordination avec leurs partenaires et leurs adversaires, dans un spectre qui va de l'usage de la force à celui de la persuasion. Une action collective, c'est une architecture mouvante de contextes de sens, plus ou moins proches ou lointains, privés ou publics, personnels ou typiques, qui articulent différentes grandeurs d'échelle spatiale et temporelle et qui sont perçus par les acteurs comme accessibles à leurs interactions ou contraignants comme des «systèmes» ou des «destins». Cette perspective de sociologie compréhensive ne se pose pas «contre» les analyses structurales, tout comme elle ne néglige pas les enquêtes macrosociologiques sur différentes instances de pouvoir ou les enquêtes macro-historiques sur des processus de longue durée. Elle s'efforce de les recadrer du point de vue des acteurs, en s'assurant qu'elles ont une pertinence dans leurs contextes d'expérience. Parfois, elle est à même de faire apparaitre des dilemmes de l'action rationnelle, des effets d'agrégation de stratégies, des fenêtres d'opportunité politique, des fronts entre blocs de réseaux ou des tensions entre logiques d'organisation, dont tous les acteurs, loin s'en faut, ne sont pas conscients. Mais le sens que les acteurs attachent à leurs propres actions, pour parler comme Weber ou la définition que les acteurs donnent de leurs propres situations, pour parler comme Thomas, reste le terrain ultime du sociologue. C'est le garde-fou le plus sûr contre les projections théoriques ou idéologiques. Les perspectives du chercheur se doivent d'être débitrices visà-vis de celles des acteurs.

6. Cette notion de «culture publique» signifie autre chose que chez Gusfield (2009 [1981]), qui menait une analyse rituelle et mythologique, à la Kenneth Burke et à la Victor Turner, de la science et du droit. Notre fil directeur est la question des conditions de possibilité de l'expérience démocratique et républicaine. 
Ces activités de faire sens ne sont donc pas réinventées à chaque fois. Elles ont un caractère typique, en partie fixées par l'institution, le droit et le décorum, en partie réglées par un jeu de prescriptions et de sanctions plus diffuses. On peut ici mentionner trois tentatives, développées en France, de ressaisir cette dimension de typicité.

a) Un effort a été engagé pour démarquer la compréhension des raisons d'agir d'une psychologie des motivations personnelles. En partant des recherches de Burke (1945) et de Mills (1940) sur le caractère situé et public des raisons et des motifs et en enchaînant sur les réflexions ethnométhodologiques sur les accounts et wittgensteiniennes sur les descriptions (Trom, 2001; Ogien, 2007), le statut des matériaux discursifs auxquels a accès l'enquêteur a été repensé. Les motifs ne sont pas des mobiles intimes, mais des façons typiques de dire, dans des contextes aux divers degrés de publicité, ce que l'on veut faire ou ce que l'on a fait. La compréhension ne s'exerce pas sur des attitudes et des opinions que les acteurs entretiendraient en leur for intérieur, mais sur des justifications, sur des relectures ou sur des annonces qui organisent l'expérience que les acteurs ont de la situation et de leurs «intentions» respectives. Les motifs sont des actes d'énonciation en public qui réordonnent l'ordre de l'interaction.

b) Dans la même veine, d'autres ont exploré des grammaires du parler en public et de l'agir en public. Les performances des acteurs doivent respecter pratiquement un certain nombre de «conditions de félicité» pour être recevables — intelligibles et acceptables - auprès de leurs auditoires, de leurs alliés et de leurs adversaires (Boltanski, 1984; Trom, 1999; Cardon et Heurtin, 1999; Lemieux, 2009). Suivre ces règles du jeu n'abolit pas le caractère ouvert, indexical et temporel des activités de faire sens - le «faire collectif» peut faire feu de tout bois, s'ouvrant des opportunités et épousant les circonstances, avec parfois une grande inventivité. Mais ces contraintes grammaticales - par exemple les «régimes de la critique, de l'opinion et du partage» (Cardon, Heurtin et Lemieux, 1995) —, si elles ne sont pas prises en compte, conduisent à la disqualification d'une dénonciation ou au discrédit d'une revendication par le public. Elles portent tant sur la pertinence des topiques d'engagement que sur la justesse des rôles de participation que sur la correction des formules d'expression (Berger, 2009).

c) D'autres ont enfin recherché des éléments de réflexion du côté d'une dramaturgie, d'une rhétorique et d'une narratologie de l'action collective, où Danto et Ricœur, Goffman, Turner et Gusfield, Burke, White et Perelman ont été les références majeures (Gusfield, 2009; Ricœur, 1983). Les acteurs puisent dans ces répertoires dramatiques de performances, rhétoriques de topoï et narratifs de récits (Cefaï, 2009), pour donner forme à leurs activités de critique, de dénonciation et de revendication. Ces modalités de mise en scène, en arguments et en récits commandant à des façons typiques de jouer, de persuader et de raconter devant des publics. Là encore, on a affaire à des actes de publicisation qui, outre qu'ils façonnent des milieux de pertinence, des paysages d'expérience et des perspectives d'action des protagonistes, s'offrent à l'assentiment ou à la critique de publics.

Mais l'identification de ces trois manières typiques de «mettre les formes » vocabulaires de motifs, grammaires du parler en public, répertoires dramatiques, rhé- 
toriques et narratifs - n'épuise pas la réflexion sur le processus de publicisation de l'action collective. Deux thématiques sont aujourd'hui à la pointe de la réflexion dans ce domaine, et pas par hasard: l'affectivité et la temporalité sont sans doute les questions les plus exigeantes, tant du point de vue de leur conceptualisation, véritables casse-tête philosophiques, que de la mise au point de dispositifs d'enquête, qui permettent d'en rendre compte. En quoi une approche pragmatiste peut-elle, là encore, nous être utile?

L'affectivité, d'abord. On a longtemps limité à la défense des thématiques de la «culture» et de l'«identité» la critique de l'analyse rationnelle et de l'analyse structurale. Mais les émotions de l'action collective ont été encore plus négligées. Elles sont récemment revenues en force dans le paysage de la recherche (Goodwin et al., 2001). Trop souvent, malheureusement, elles l'ont été à travers le schéma de la manipulation des apparences - l'impression management — dans des stratégies de communication publique, d'organisation de rassemblements ou de communication à des auditoires. La question avait déjà été soulevée en France, d'un point de vue théorique, par des auteurs comme Livet $(2002)$ et Thévenot $(1994,1997)$ ou Paperman et Ogien (1995), qui avaient fait un pas au-delà de l'opposition entre raison et passion, cognition et affection. Elle l'avait été bien avant, encore, quand Park invitait à une météorologie des atmosphères publiques, avec leurs variations climatiques et leurs mouvements barométriques, et liait ce souci pour la Stimmung à l'enquête sur l'opinion publique et sur le comportement collectif. Au lieu de techniques de gestion des sentiments des autres, les passions et les affects retrouvent leur dimension de "passibilité à des événements» (Quéré, 2003) ou d'affection par des situations (Cefaï et Lafaye, 2001): de l'émoi, du malaise ou de la panique, de la liesse, du dégoût ou de l'enthousiasme, qui touchent au moral, aux affectivités et aux sensibilités collectives, en situation. Le sens du bien, du droit et de la justice, le sens de l'honneur, la passion de l'égalité ou la haine de classe sont avant tout des sentiments moraux. Toute mobilisation collective est scandée par des épreuves d'indignation et de révolte, de solidarité ou de débandade, de joie et de déception - l'apathie elle-même ne va pas sans pathos. Les émotions, qu'elles restent encloses dans un cercle privé ou qu'elles se mettent à avoir une portée collective et une résonance publique, sont ce qu'il y a de plus difficile à décrire: la fierté bafouée, la soif de revanche, le souci de vérité, le désir de liberté, l'orgueil national, la haine raciale... et plus simplement, les manifestations expressives dans des situations données, avec tout ce qu'elles peuvent avoir d'insaisissable. L'ethnographie doit alors gagner une qualité littéraire, sans pour autant renoncer à la discipline de l'observation et de la description. Breviglieri est peut-être le plus sensible à certaines de ces épreuves affectives (2009).

Plus radicalement, il ne s'agit pas de simplement réhabiliter un «objet» jusque-là négligé par l'enquête ou l'analyse, mais bien de transformer la définition de l'action collective. L'affectivité n'est pas un thème parmi d'autres. Elle est ce qui fait qu'il y a expérience, tant perceptive que cognitive ou morale. Elle n'est pas une coloration d'états de fait ou d'états de conscience. Elle est ce qui garantit notre contact avec les autres et avec les choses, et ce qui fait tenir ensemble les situations en nous y situant. L'action 
collective n'est pas toute dans l'agir, mais aussi dans le pâtir et le compatir. Elle a une dimension d'affection et de passion collective. Avant d'être propulsée par des visées stratégiques, les membres qui s'engagent sont affectés par les situations qu'ils contribuent à définir et à maîtriser; ils sont "passibles», exposés à des événements qui les bouleversent et les déroutent, remaniant leurs critères de compréhension et redéployant leurs horizons d'intelligibilité. En deçà des stratégies rationnelles ou des déterminations structurales, l'action collective se laisse ébranler par des événements, qui parfois n'affectent que des particuliers, qui parfois prennent une autre ampleur et concernent des gens bien au-delà de ceux qui sont directement touchés (Dewey, 1927), les poussant à se former une expérience commune, à s'indigner de concert et à se mobiliser en public. Ces dynamiques de ré-articulation des champs d'expérience et des horizons d'attente (Koselleck, 1997) altèrent le sens de ce qui est «capital», «ressource», «contrainte» ou «opportunité». Une bonne explication compréhensive ne peut pas jongler avec ces catégories comme si elles étaient porteuses d'un sens stable, à la validité trans-situationnelle. Elles relèvent d'une grammaire de l'action, engagent des dispositions des acteurs et des dispositifs d'action. Elles requièrent des régimes d'expérience. Le terme d'expérience peut ici être entendu de trois façons, où à chaque fois, nous nous trouvons affectés de façon différente. L'expérience comme épreuve esthétique: les sens affectifs (pathos) et esthétiques (aisthesis) sont ce qui, en deçà des raisonnements et des jugements, nous donnent accès au monde, des charnières de l'expérience qui font que nous sommes en prise sur les situations parce qu'elles ont une emprise sur nous. L'expérience comme expérimentation pratique: la reconnaissance du réel est éprouvée par les tests que l'on peut faire subir à des situations et aux résultats auxquels nous devons nous soumettre, accroissant notre capacité d'action en nous pliant à une réalité transcendante. L'expérience comme échange interactif: notre accès à la vérité, au bien et au droit n'est jamais solitaire, mais passe par des interactions avec les autres et avec les choses, par l'intermédiaire de procès de coopération et de communication, qui prennent parfois la forme de véritables délibérations, où nous nous exposons aux points de vue des autres. Notre expérience de l'engagement a une dimension sensuelle, expérimentale et interactionnelle. Et les catégories de l'action collective (capital, ressource, contrainte, opportunité...) sont à chaque fois à ressaisir depuis leur ancrage dans les contextes de sens des acteurs.

La temporalité, ensuite. L'enquête doit se rendre sensible à l'émergence temporelle des actions collectives. Elle doit choisir des terrains qui lui assurent l'accès à de tels corpus de matériaux. Plusieurs trains de recherche sont déjà allés dans ce sens. Il peut s'agir de trajectoires biographiques, de personnes ou d'organisations: Fillieule (2001, 2005) a engagé un programme de recherches qui essaie de coupler analyse de cohortes d'âges dans des organisations et analyse de trajectoires d'engagement et de désengagement. Jasper (1997), sur les tireurs d'alarme dans des industries à risque, ou Auyero (2003), sur une piquettera en Patagonie et une émeutière à Santiago, ont également replacé les accidents de l'expérience morale, incarnée dans des cours existentiels, au cour de la mobilisation. Dans un livre crucial, peu lu par les sociologues, sur les tra- 
jectoires d'ouvriers d'un quartier turinois, Gribaudi (1987) a suivi des cohortes d'amis et de parents sur plusieurs générations, en les réinscrivant par rapport à des projets familiaux et personnels de migration urbaine et d'ascension sociale et en reconstituant leurs réseaux égocentrés: leur passage du socialisme au fascisme est expliqué à partir de choix biographiques dans des environnements politiques en mutation. Ce modèle de flux et de strates d'expérience qui s'entrecroisent, corrélativement à des épreuves, successives ou simultanées, de socialisation, pourrait enrichir l'analyse biographique des mobilisations.

Là encore, les approches pragmatiques et pragmatistes ont ouvert un certain nombre de chantiers. L'attention a été déplacée de ces carrières personnelles ou organisationnelles vers des carrières de problèmes publics, autour desquelles s'articulent différentes configurations d'arènes publiques. Chateauraynaud et Torny (1999) le font à leur façon, quand ils examinent les dossiers des prions, de l'amiante et du nucléaire. Ils constituent ainsi des séries longitudinales d'archives qu'ils analysent ensuite au moyen des logiciels Prospero ou Marlowe. Terzi (2003) a de son côté travaillé — sans traitement informatique - sur un énorme corpus de presse concernant l'affaire de la spoliation de l'argent des juifs en Suisse pendant la Seconde Guerre mondiale. Il s'en est tenu exclusivement, en mettant entre parenthèses toute entreprise d'histoire sociale, à rendre compte de la dynamique interne de la controverse, de l'organisation de séquences de reformulation et de dispute, de l'émergence de nouvelles catégories et intrigues (Terzi et Bovet, 2005) — montrant la constitution de cette affaire publique sur plusieurs décennies. D’autres travaux, fondés sur l'enquête ethnographique, autant que sur l'enquête sur documents ou par entretiens, ont également restitué la genèse d'arènes publiques, dans toute leur complexité: celui de Lolive (1997 et 1999) sur les contestations contre le TGV Méditerranée ou celui de Dodier (2003) sur l'histoire des mobilisations autour de l'épidémie du sida. Mais la temporalité peut encore être déconnectée du support biographique des personnes et de la moyenne durée des affaires publiques. Dans le court terme, l'enquête peut suivre la temporalité de moments d'épreuve qui provoquent des mobilisations soudaines — qu'il s'agisse d'événements comme celui de la profanation du cimetière de Carpentras (Barthélémy, 1991) ou de disputes, comme les affaires Calas et du Chevalier de la Barre (Claverie, 1994 et 1998). Elle peut encore prendre la forme des controverses que les étudiants de l'École des Mines et de Sciences Po étudient aujourd'hui, emboîtant le pas à l'entreprise de Latour depuis son étude sur la «pasteurisation de la France» (Latour, 1984) — avec, dans l'étude des dispositifs d'action publique, un recroisement entre les conceptions de sociologie des organisations, des cités de justification, et des sciences et des techniques (Lolive, 1999).

La reconnaissance de la passibilité des acteurs et le réarmement de leurs capacités morales et politiques ont permis de rapatrier en sciences sociales la question de la raison pratique, à l'encontre d'une conception étroite de la stratégie. La question de la temporalité des actions collectives en sort transformée. L'action collective ne se «construit » pas seulement en suivant des plans (Quéré et Pharo, 1990; Ladrière et al., 
1993). Elle se fait en pilotant à vue dans l'embrouillamini des circonstances, s'efforce de lier des contingences qui lui arrivent et qu'elle subit, tente de retracer et de relancer des lignes d'action en faisant face à des situations qui lui échappent. Et elle n'est pas simplement une affaire de repérage cognitif dans le monde: elle est traversée par des épreuves de droit et de devoir, d'obligation et de responsabilité, vis-à-vis des humains et des non-humains - dieux ou bêtes, lieux ou choses. En outre, elle se déploie temporellement, ce qui signifie que la dissection de l'action collective en éléments, structurels ou conjoncturels, ne nous livre pas son secret. Elle est vécue parfois comme une routine qui suit son rythme de croisière, parfois sous la figure de l'événement qui fait irruption et qu'il faut affronter. Le cours de l'action n'est pas uniforme. Il est semé d'embûches, interrompu par des péripéties et des rebondissements, il expose à des machinations et à des traquenards, est relancé par des manœuvres et des projets. Souvent, il prend des directions imprévues, déroute les expectatives de ses agents, bifurque en plusieurs possibilités, impose de peser le pour et le contre. Il donne le sentiment d'accélérer sans laisser le temps de réfléchir ou d'enfermer au contraire dans un piège sans issue. Il prend l'allure d'une avenue ou au contraire celle d'une impasse. En recourant aux vertus antiques du courage (virtú), de l'intelligence située (metis) et de la prudence pratique (phronesis), ceux qui portent et supportent l'action collective peuvent saisir leur chance, infléchir le destin en bonne ou mauvaise fortune, déceler dans l'existant l'horizon du possible, tout en sachant qu'ils n'anticiperont jamais tout à fait les effets de ce qu'ils entreprennent. Le prix de la liberté d'agir est dans l'imprévisibilité de ses résultats et de ses conséquences - ce que constatent de plus en plus les enquêtes sur les mouvements sociaux (Giugni et al., 1999). Et le savoir-faire des leaders d'organisations réside peut-être autant dans cet art du jugement pratique que dans une technologie du management.

Ces différents éléments bouleversent la compréhension que nous pouvons avoir de ce qui est en jeu dans une action collective, au-delà du jeu des forces et des intérêts: un sens de la réalité, du droit et de la justice (Cottereau, 1992 et 1999). L'entrée principale dans le terrain est alors celle de l'expérience, particulière, collective et publique. L'existence d'une «culture publique», qui émerge à travers le travail de mobilisation et qui, au-delà, se stabilise dans un certain nombre de médiations conventionnelles et institutionnelles, autorise la constitution d'une expérience collective et l'exercice d'un jugement de sens commun. La «culture publique» n'est plus seulement alors une «boîte à outils »: elle pré-articule les structures d'horizon de la vie collective, lui fournit ses matériaux d'expérience et rend possible l'articulation d'une arène publique où soient disponibles les critères du vrai et du faux, du réel et de l'imaginaire, de l'existant et du possible, du juste et de l'injuste, du droit et du tort, du légitime et de l'illégitime. L'action collective trouve nécessairement ses appuis dans cette «culture publique» qui, tout en étant partagée par ses membres, leur fournit des options de prise de parti, leur attribue des places dans des arènes de bataille et leur indique les bonnes formes expressives. Elle peut s'engager dans la logique du groupe de pression qui joue la carte du lobbying ou dans celle de la confrontation guerrière où l'emporte le plus fort — «force» et 
«intérêt» renvoient à des grammaires de la confrontation et de la négociation, qui ont aussi leurs règles du jeu ${ }^{7}$. Elle peut se rapprocher d'une conception de la délibération publique, quand l'argumentation, l'enquête ou l'expérimentation fournissent des faits et des arguments visant à convaincre rationnellement et raisonnablement: de nouvelles modalités de participation des citoyens et de coopération avec élus et experts sont ainsi revendiquées.

Mais la focalisation de l'enquête sur ces modes d'existence de la «culture publique» ne doit pas faire perdre de vue la multiplicité des épreuves de la vie quotidienne et leur résonance dans l'expérience collective, sur différentes scènes publiques, qui contribuent également à articuler l'action collective. Les «mouvements sociaux», pour ou contre l'avortement, l'euthanasie, la biogénétique, la parité entre hommes et femmes, les droits des animaux ou la protection de l'environnement formulent leurs dénonciations et leurs revendications en s'appuyant sur cet arrière-fond. Ce faisant, ils s'identifient, se coordonnent et co-agissent, et, en s'adressant aux affectivités, aux sensibilités et aux entendements de leurs publics, ils font bouger les critères de l'expérience des "particuliers», dans la sphère de la relation intime ou du colloque personnel. Ils redéfinissent des situations, réélaborent des visions du monde et re-déploient des convictions et des identités. Les mouvements sociaux sont des analyseurs, des catalyseurs et des accélérateurs de la formation de cette «culture publique» et, en contrepoint, de ce que l'on appelait autrefois la transformation des mœurs, des usages et des coutumes.

\section{EN GUISE DE CONCLUSION}

Nous espérons, par ce trop bref tour d'horizon, avoir donné une idée de la fécondité, dans la pluralité, de quelques-unes des approches pragmatiques et praxéologiques, à l'occasion recadrées dans une perspective pragmatiste, pour l'étude de l'action collective. On peut légitimement se demander pourquoi leur réception a été aussi faible. Plusieurs réponses s'esquissent. D'abord, une représentation étroite des mobilisations collectives a conduit à se focaliser sur les dilemmes de la rationalité ou sur les mouvements sociaux, interdisant de considérer d'autres objets - alors qu'ils proliféraient, par exemple dans le domaine de la sociologie des sciences et des techniques. Ensuite, l'agenda de recherche se partageait autour de grands modèles. En langue française, les héritages tourainien, d'abord, puis bourdieusien, se sont taillé la part du lion, tandis qu'à partir des années 1990, le Groupe d'étude et de recherche sur les mutations du militantisme a fait en France un travail important de transplantation et d'acclimatation des recherches «nord-américaines». La prime aux modèles légitimes a joué à plein, d'autant que les auteurs ici présentés se souciaient assez peu de la réception et de la reprise de leurs recherches dans le domaine de la sociologie des mobilisations collectives: leurs objets théoriques et empiriques, leurs partenaires et leurs adversaires de

7. Les rapports de force, centraux chez Callon et Latour, avaient pratiquement disparu dans le programme de recherche sur les formes de justification en public du GSPM, qui prenait leur contrepied. Ils ont été réintroduits par Boltanski et Chiapello (1999), mais on les trouve aussi bien sous d'autres formes dans les recherches sur la «violence politique» ou sur les «asymétries de prise» (Chateauraynaud, 2006 et 2008). 
controverse étaient ailleurs. Ce n'est somme toute que récemment que leurs idées ont commencé à circuler davantage et à être appliquées par d'autres chercheurs que leurs étudiants directs. En France, cette diffusion est facilitée par le fait que les inimitiés d'école, les ostracismes et les aversions s'atténuent avec le temps. Le changement des générations devrait permettre aux enjeux de l'enquête et de l'analyse de primer sur les loyautés personnelles et les attachements dogmatiques.

\section{RÉSUMÉ}

En présentant un ensemble de recherches «pragmatiques » ou «praxéologiques » qui ont eu cours en France depuis les années 1980, et en les augmentant de quelques idées héritées du pragmatisme nord-américain, l'auteur s'interroge sur leur pertinence pour une sociologie des mobilisations collectives. L'article s'ordonne autour de quatre points.

1. Le développement d'une sociologie des régimes d'action et de justification, d'une part, et de démarches herméneutiques ou grammaticales, d'autre part, complexifie les modélisations structuraliste et rationaliste de l'action.

2. La réactivation de la notion de «public», empruntée à Dewey, en contrepoint d'une sociologie des problèmes publics, permet de poser des questions sur le politique que les approches en termes de processus, de marché ou de champ politique avaient négligées.

3. L'étude des réseaux et des organisations les conçoit comme des arènes d'expérience et d'action, articulées par des agencements d'objets, dans lesquelles les acteurs s'engagent selon des modalités hybrides. Elle fait apparaître des grammaires plurielles de la communauté, de l'acteur et du collectif.

4. L'approche instrumentale ou stratégique de la culture, entendue comme répertoire de ressources, est enrichie par une conception de la culture publique, centrée sur la formation d'expériences individuelles et collectives. Elle s'appuie sur des réflexions autour des notions d'affectivité et de temporalité.

\section{ABSTRACT}

The author presents the "pragmatic" or "praxeological" research underway in France since the 1980 , includes some concepts passed down from North American pragmatism, and queries the overall relevance for a sociology of collective mobilizations. Four main points are discussed:

1. The development of a sociology of plans of action and justification in tandem with the hermeneutical or grammatical approaches complicates the structuralist and rationalist modelings of action.

2. Reactivation of the notion of "public", borrowed from Dewey, in contrast to a sociology of public problems, elicits political questions neglected by the approaches focused on processes, the market and the political field.

3. The study of networks and organizations conceives them as arenas of experience and action articulated by juxtapositions of objects in which the actors engage according to hybrid modalities. Grammars of the community, the actor and the collective emerge.

4. The instrumental or strategic cultural approach, seen as a repertoire of resources, is enriched by a notion of public culture that shapes individual and collective experiences. Affectivity and temporality are explored as underpinning concepts. 


\section{RESUMEN}

Al presentar un conjunto de investigaciones "pragmáticas" o "praxeológicas" que se llevaron a cabo en Francia desde los años ochenta, y complementándolas con algunas ideas heredadas del pragmatismo norteamericano, el autor se pregunta sobre su pertinencia para una sociología de la acción colectiva. El artículo se ordena a partir de cuatro puntos.

1. El desarrollo de una sociología de los regímenes de acción y de justificación, por una parte, $y$ los planteamientos hermenéuticos o gramaticales, por otra parte, complican los modelos estructuralistas y racionalistas de la acción.

2. La reactivación de la definición "de lo público", tomada de Dewey, en contraste con una sociología de problemas públicos, permite plantear preguntas sobre "la política" que los enfoques que se basan en los procesos, en el mercado o en el campo político no habían considerado.

3. El estudio de las redes y de las organizaciones los concibe como arenas de experiencia y de acción, articuladas por yuxtaposición de objetos, en las cuales los actores se involucran de acuerdo a modalidades híbridas. Surgen gramáticas plurales de la comunidad, del actor y del colectivo.

4. El enfoque instrumental o estratégico de la cultura, comprendido como un directorio de recursos, se enriquece por una concepción de la cultura pública, centrada en la formación de experiencias individuales y colectivas. Este enfoque se basa también en reflexiones en torno a las nociones de afectividad y temporalidad.

\section{BIBLIOGRAPHIE}

Arendt, H. (1972), Du mensonge à la violence, Paris, Calmann-Lévy.

Auray, N. (2002), «De l'éthique à la politique: l'institution d'une cité libre», Multitudes, no 8.

Auyero, J. (2003), Contentious Lives: Two Argentine Women, Two Protests, and the Quest for Recognition,

Durham et Londres, Duke University Press.

Bаввот, J. (2002), Les malades en mouvements. La médecine et la science à l'épreuve du sida, Paris, Balland.

BarthéLÉMY, M. (1992), «Événement et espace public: l'affaire Carpentras», Quaderni, 18, p. 125-140.

Berger, M. (2009), Répondre en citoyen ordinaire. Enquête sur les «engagements profanes» dans un dispositif d'urbanisme participatif à Bruxelles, Bruxelles, Thèse de doctorat de l'ULB.

Boltanski, L. (1990 [1984]), «La dénonciation », in L’Amour et la justice comme compétences, Paris, Métailié. Boltanski, L. et L. Thévenot (1991), De la justification. Les économies de la grandeur, Paris, Gallimard. Boltanski, L. et E. Chiapello (1999), Le nouvel esprit du capitalisme, Paris, Gallimard.

Breviglieri, M. (2009), «L'insupportable. L'excès de proximité, l'atteinte à l'autonomie et le sentiment de violation du privé», in Breviglieri, M., Lafaye, C., et D. Trom (dir.), Compétences critiques et sens de la justice, Paris, Économica.

Burke, K. (1945), «Introduction: The Five Key Terms of Dramatism», in A Grammar of Motives, Berkeley, University of California Press, p. 15-18.

Callon, M. et V. Rabeharisoa (1999), Le pouvoir des malades. L'Association française contre les myopathies et la recherche, Paris, Presses de l'École des Mines.

Callon, M., P. Lascoumes et Y. Barthe (2001), Agir dans un monde incertain. Essais sur la démocratie technique, Paris, Seuil.

Cardon, D. et J.-P. Heurtin (1999), «La critique en régime d'impuissance. Une lecture des indignations des auditeurs de France-Inter», in François, B. et É. Neveu (dir.), Espaces publics mosaïques. Transformations de l'espace public, Rennes, PUR.

Cardon, D., J.-P. Heurtin et C. Lemieux (1995), «Parler en public», Politix, 31, p. 5-19. 
Cefaï, D. (2001), «Les cadres de l'action collective. Définitions et problèmes», in Cefaï, D. et D. Trom, Les formes de l'action collective. Mobilisations dans les arènes publiques. p. 51-97.

CEFAï, D. (2002), «Qu'est-ce qu'une arène publique? Quelques pistes pour une approche pragmatiste», in

Cefaï, D. et I. Joseph, L'héritage du pragmatisme, p. 51-82.

CEFAï, D. (2007), Pourquoi se mobilise-t-on? Les théories de l'action collective, Paris, La Découverte.

Cefaï, D. (2009), «Boire ou conduire, il faut choisir! La fabrique des problèmes publics», postface à

J. Gusfield, La culture des problèmes publics, Paris, Économica, p. 219-318.

Cefaï, D. et I. Joseph (dir.) (2002), L'Héritage du pragmatisme, La Tour d'Aigues, Éditions de l'Aube.

CеFAï, D. et D. Тrom (dir.) (2001), Les formes de l'action collective. Mobilisations dans des arènes publiques, Paris, Éditions de l'Ehess, «Raisons pratiques», vol. nº 12.

Cefaï, D. et C. LAFAye (2001), «Lieux et moments d'une mobilisation collective. Le cas d'une association de quartier», in CеFAї, D. et D. Trom, Les formes de l'action collective. Mobilisations dans des arènes publiques, p. 195-228.

Chateauraynaud, F. (2006), «Les asymétries de prise. Des formes de pouvoir dans un monde en réseaux», GSPR-EHESS.

Chateauraynaud, F. (2008), «Des disputes ordinaires à la violence politique. L'analyse des controverses et la sociologie des conflits», GSPR-EHESS.

Chateauraynaud, F. et D. Torny (1999), Les sombres précurseurs. Une sociologie pragmatique de l'alerte et $d u$ risque, Paris, Éditions de l'Ehess.

Claverie, E. (1994), «Procès, affaire, cause: Voltaire et l'innovation critique», Politix, 26, p. 76-86.

Claverie, E. (1998), «La naissance d'une forme politique: l'affaire du Chevalier de la Barre», in Roussin, P. (dir.), Critique et affaires de blasphème à l'époque des Lumières, Paris, Honoré Champion.

Clemens, E. (1997), The People's Lobby: Organizational Innovation and the Rise of Interest Group Politics in the United States 1890-1925, Chicago, University of Chicago Press.

Conein, B et L. Thévenot (dir.) (1997), Cognition et information en société, Paris, Éditions de l'Ehess, «Raisons Pratiques», $\mathrm{n}^{\circ} 8$.

Corcurf, P. (1991), Constructions du mouvement ouvrier. Activités cognitives, pratiques unificatrices et conflits dans un syndicat de cheminots, Paris, Thèse de doctorat EHESS.

Cottereau, A. (1992), «Esprit public et capacité de juger. La stabilisation d'un espace public en France aux lendemains de la Révolution », in Cottereau, A. et P. Ladrière (dir.), Pouvoir et légitimité, Paris, Éditions de l'EHess, «Raisons pratiques», n 3, p. 239-273.

Cottereau, A. (1999), «Dénis de justice, dénis de réalité: remarques sur la réalité sociale et sa dénégation», in Dulong, R. et P. Gruson (dir.), L’Expérience du déni, Paris, Éditions de la MSH, p. 159-178.

De Munck, J. et B. Zimmermann (2009), La liberté au prisme des capacités, Paris, Éditions de l'EHESS («Raisons pratiques», $\left.\mathrm{n}^{\circ} 18\right)$

Dewey, J. (1993 [1938]), Logique. La théorie de l'enquête, Paris, PUF.

Dewey, J. (2003 [1927]), Le public et ses problèmes, Pau, Publications de l'Université de Pau, Tours, Farrago/ Leo SCheER (trad. fr. et présentation J. ZASK).

Diani, M. (1992), «The Concept of Social Movement», Sociological Review, 40, p. 1-25.

Diani, M. et D. McAdam (dir.) (2003), Social Movements and Networks, Oxford, Oxford University Press. Dodier, N. (1991), «Agir dans plusieurs mondes», Critique, 529-530, p. 428-458.

Dodier, N. (1993), «Les appuis conventionnels de l’action. Éléments de pragmatique sociologique», Réseaux, 62, p. 65-85.

Dodier, N. (1995), Les hommes et les machines. La conscience collective dans les sociétés technicisées, Paris, Éditions Métailié.

Dodier, N. (2003), Leçons politiques de l'épidémie de sida, Paris, Éditions de l'Ehess.

Dordy, É. (2002), La vulnérabilité du sujet politique. Régimes de proximité dans les arènes d'engagement public, Thèse de doctorat, Paris, EHess.

Dupuy, J.-P. et P. Livet (dir.) (1997), Les limites de la rationalité. Rationalité, éthique et cognition, Paris, La Découverte. 
Eliasoph, N. et P. Lichterman (2003), «Culture in Interaction », American Journal of Sociology, 108, 4, p. 735-794.

Emirbayer, M. et J. Goodwin (1994), «Network Analysis, Culture, and the Problem of Agency», American Journal of Sociology, 99, 6, p. 1411-1454.

Fillieule, O. (2001), «Post-scriptum: Propositions pour une analyse processuelle de l'engagement individuel», Revue politique de science politique, 51, 1-2, p. 199-217.

Fillieule, O. (2005), «Temps biographique, temps social et variabilité des rétributions», in Fillieule, O. (dir.), Le désengagement militant, Paris, Belin, p. 17-47.

Follett, M. P. (1998 [1918]), The New State: Group Organisation, the Solution of Popular Government, Philadelphie, Pennsylvania University Press.

Friedberg, E. (1992), «Les quatre dimensions de l'action organisée», Revue française de sociologie, 33, 4, p. 531-557.

Giugni, M., D. McAdam et C. Tilly (dir.) (1999), How Social Movements Matter, Minneapolis, University of Minnesota Press.

Goffman, E. (1991 [1974], Les cadres de l'expérience, Paris, Minuit.

Gonzalez, P. (2009), Voix des textes, voies des corps. Une sociologie du protestantisme évangélique, Fribourg et Paris, Thèse de sociologie de l'Université de Fribourg et de l'EHESS.

Goodwin, J. et J. JASPER (2004 [1999]), «Caught in a Winding, Snarling Vine: The Structural Bias of Political Process Theory», in Id. (dir.), Rethinking Social Movements: Structure, Meaning, and Emotion, Lanham, Rowman \& Littlefield, p. 3-30.

Goodwin, J., J. Jasper et F. Polletta (dir.) (2001), Passionate Politics: Emotions and Social Movements, Chicago, University of Chicago Press.

Gribaudi, M. (1987), Itinéraires ouvriers. Espaces et groupes sociaux à Turin au début du Xx $x^{e}$ siècle, Paris, Éditions de l'Ehess.

Gusfield, J. (1981), «Social Movements and Social Change: Perspectives on Linearity and Fluidity», in Kriesberg, L. (dir.), Social Movements, Conflict, and Change, 4, p. 317-339.

Gusfield, J. (2009 [1981]), La culture des problèmes publics, Paris, Économica.

Ion, J. (2001), L’Engagement au pluriel, Saint-Étienne, Presses de l'Université de Saint-Étienne.

JAMES W. (2007 [1912]), Essais d'empirisme radical, Paris, Flammarion.

JAsper, J. (1997), The Art of Moral Protest: Culture, Biography, and Creativity in Social Movements, Chicago, University of Chicago Press.

JAsper, J. (2006), Getting Your Way, Chicago, University of Chicago Press.

Joseph, I. (2007), L'Athlète moral et l'enquêteur modeste, Paris, Économica.

Koselleck, R. (1997), L'Expérience de l'histoire, Paris, Gallimard et Seuil.

LAdrière, P., P. Pharo et L. Quéré (dir.), La théorie de l'action, Paris, Éditions du CNRS, 1993.

LAFAYE, C. (1996), Sociologie des organisations, Paris, Armand Colin.

LAFAYE, C. (2001), «L'ancrage de proximité en politique(s): mobilisations locales et contestations de l'action publique», Revue de l'Institut de Sociologie, Université Libre de Bruxelles, 1-4, p. 109-112.

Lamont, M. et L. Thévenot (dir.) (2000), Rethinking Comparative Cultural Sociology, Cambridge University Press/Éditions de la MSH.

Latour, B. (1984), Les microbes, guerre et paix, suivi de Irréductions, Paris, Métailié.

Latour, B. (1999), Politiques de la nature. Comment faire entrer les sciences en démocratie, Paris, La Découverte.

Latour, B. (2008), «Le fantôme de l'esprit public», in Lippmann, W., Le public fantôme, Paris, Demopolis, p. 3-44.

Lemieux, C. (2009). Le devoir et la grâce, Paris, Économica.

Lichterman, P. (1996), The Search for Political Community: American Activists Reinventing Commitment, New York, Cambridge University Press.

Lichterman, P. (2005), Elusive Togetherness: Church Groups Trying to Bridge America's Divisions, Princeton, Princeton University Press. 
Livet, P. (2002), Émotions et rationalité morale, Paris, PUF.

Lolive, J. (1997), «La montée en généralité pour sortir du Nimby. La mobilisation associative contre le TGV Méditerranée», Politix, 39, p. 109-130.

Lolive, J. (1999), Les contestations du TGV Méditerranée, Paris, L'Harmattan

Lolive, J. (2006), «Des forums hybrides à l'esthétisation des espaces publics», Cahiers de géographie du Québec, 50, 140, p. 151-171.

McAdam, D., S. Tarrow et C. Tilly (2001) Dynamics of Contention, Cambridge, Cambridge University Press.

MeAd, G.-H. (2006 [1934]), L'Esprit, le soi et la société, Paris, PUF.

Melucci, A. (1996), Challenging Codes, Cambridge, Cambridge University Press.

Micoud, A. (2007), «De l'expert-militant à l'être vivant sensible», in "Esthétique et espace public», Cosmopolitiques, $\mathrm{n}^{\circ}$ 15, p. 121-134.

Mische, A. (2008), Partisan Publics: Communication and Contention Across Brazilian Youth Activist Networks, Princeton, Princeton University Press.

Ogien, A. (2007), Les formes sociales de la pensée. La sociologie après Wittgenstein, Paris, Armand Colin.

OrléAn, A. (dir.) (1994), Analyse économique des conventions, Paris, PUF.

Paperman, P. et R. Ogien (dir.) (1995), La couleur des pensées, Paris, Éditions de l'Ehess, « Raisons pratiques $", n^{\circ} 6$.

Pattaroni, L. (2001), «Le geste moral. Perspective sociologique sur les modalités du vivre-ensemble», Carnets de bord, $\mathrm{n}^{\mathrm{o}}$ 2, p. 67-77.

Pattaroni, L. (2005), Politique de la responsabilité. Promesses et limites d'un monde fondé sur l'autonomie, Paris-Genève, Thèse de doctorat EHESS et Université de Genève.

Pharo, P. (1985), Le civisme ordinaire, Paris, Méridiens-Klincksieck.

Polletta, F. (2002), Freedom is an Endless Meeting: Democracy in American Social Movements, Chicago, University of Chicago Press.

QuéRÉ, L. (1990), «L'opinion: l'économie du vraisemblable. Introduction à une approche praxéologique de l'opinion publique», Réseaux, n 43, p. 33-58.

QuÉRÉ, L. (1992), «L'espace public: de la théorie politique à la métathéorie sociologique», Quaderni, 18.

QUÉRÉ, L. (1995), «L'espace public comme forme et événement», in JosEPH, I. (dir.), Prendre place. Espace public et culture dramatique, Paris, Plan Urbain, 1995, p. 93-110.

Quéré, L. (1996), «L'espace public comme lieu de l'action collective», Mana, 2.

Quéré, L. (2003), «La structure de l'expérience publique d'un point de vue pragmatiste», in L'Héritage du pragmatisme, in CEFAÏ, D. et I. Joseph.

Quéré, L. et P. Pharo (dir.) (1990), Les formes de l'action, Paris, Éditions de l’Ehess, 1990, « Raisons pratiques», $\mathrm{n}^{\circ} 1$.

Quéré, L. (1997), «La situation négligée», Réseaux, 85, p. 163-192.

Rosanvallon, P. (2006), La contre-démocratie. La politique à l'âge de la défiance, Paris, Seuil.

Stavo-Debauge, J. et D. Trom (2004), «Le pragmatisme et son public à l'épreuve du terrain. Penser avec Dewey contre Dewey», in Karsenti, B. et L. Quéré (dir.), La croyance et l'enquête. Aux sources du pragmatisme, Paris, Éditions de l'EHESS ( «Raisons pratiques», $\mathrm{n}^{\circ} 15$ ).

TAYlor, V. (2005 [1989]), «La continuité des mouvements sociaux. La mise en veille du mouvement des femmes", in Fillieule, O. (dir.), Le désengagement militant, p. 229-250.

Terzi, C. (2003), Qu'avez-vous fait de l'argent des juifs?, Paris, Thèse de doctorat EhEss, Fribourg, Université de Fribourg.

Terzi, C. et A. Bovet (2005), «La composante narrative des controverses politiques et médiatiques. Pour une analyse praxéologique des actions et des mobilisations collectives», Réseaux, 132, 4, p. 111-132.

Thévenot, L. et M. Breviglieri (dir.) (2010), Politiques au regard du proche, à paraître à Paris, Économica.

Thévenot, L. (1993), «La trame des organisations», in T. Globokar (dir.), Entreprise, société, communauté, Paris, Autrement. 
Thévenot, L. (1999), «Faire entendre une voix. Régimes d'engagement dans les mouvements sociaux», Mouvements, 3, p. 73-82.

Thévenot, L. (2006), L'Action au pluriel. Sociologie des régimes d'engagement, Paris, La Découverte.

Tilly, C. (1995), «To Explain Political Process», American Journal of Sociology, 100, 6, p. 1594-1610.

Tilly, C. (2003), The Politics of Collective Violence, Cambridge, Cambridge University Press.

Trom, D. et P. Laborier (dir.) (2003), Historicités de l'action publique, Paris, PUF.

Твом, D. (1999), «De la réfutation de l'effet Nimby considérée comme une pratique militante. Notes pour une approche pragmatique de l'activité revendicative», Revue française de science politique, 1, p. 31-50.

Твом, D. (2001), «Grammaire de la mobilisation et vocabulaires de motifs: origines et actualité d'une perspective", in Cefaї, D. et D. Trom, Les formes de l'action collective. Mobilisations dans les arènes publiques, p. 99-134. 